Research Paper

\title{
Effects of Mild Chronic Intermittent Cold Exposure on Rat Organs
}

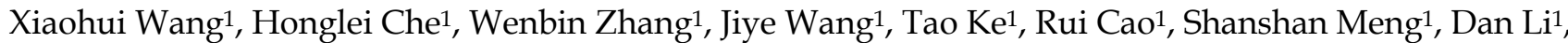 \\ Ouyang Weiming ${ }^{2}$, Jingyuan Chen ${ }^{1 凶}$, Wenjing Luo ${ }^{1 凶}$ \\ 1. Department of Occupational \& Environmental Health and the Ministry of Education Key Lab of Hazard Assessment and Control in Special \\ Operational Environment, School of Public Health, Fourth Military Medical University, Xi'an 710032, China \\ 2. Immunology Program, Memorial Sloan-Kettering Cancer Center, New York, New York 10065, USA
}

$\triangle$ Corresponding authors: Wenjing luo and Jingyuan chen, Department of Occupational and Environmental Health, School of Public Health Fourth Military Medical University, 17 Changlexi Street, Xi'an, China Phone: +86 29 84774863; Fax: +86 29 84774862; Email: luowenj@fmmu.edu.cn, or jy_chen@fmmu.edu.cn.

(c) 2015 Ivyspring International Publisher. Reproduction is permitted for personal, noncommercial use, provided that the article is in whole, unmodified, and properly cited. See http://ivyspring.com/terms for terms and conditions.

Received: 2015.03.18; Accepted: 2015.06.26; Published: 2015.08.08

\begin{abstract}
Cold adaptation is a body's protective response to cold stress. Mild chronic intermittent cold (CIC) exposure has been used to generate animal models for cold adaptation studies. However, the effects of mild $\mathrm{ClC}$ exposure on vital organs are not completely characterized. In the present study, we exposed rats to mild CIC for two weeks, and then measured the body weights, the weights of brown adipose tissue (BAT), the levels of ATP and reactive oxygen species (ROS) in the brains, livers, hearts, muscles and BATs. Rats formed cold adaptation after exposure to $\mathrm{CIC}$ for two weeks. Compared to rats of the control group that were hosted under ambient temperature, rats exposed to mild $\mathrm{CIC}$ showed a lower average body weight, but a higher weight of brown adipose tissue (BAT). Rats exposed to ClC for two weeks also exhibited higher levels of ATP and ROS in all examined organs as compared to those of the control group. In addition, we determined the expression levels of cold-inducible RNA binding protein (Cirbp) and thioredoxin (TRX) in rat tissues after 2 weeks of $\mathrm{CIC}$ exposure. Both Cirbp and TRX were increased, suggesting a role of these two proteins for establishment of cold adaptation. Together, this study reveals the effects of mild $\mathrm{CIC}$ exposure on vital organs of rats during $\mathrm{CIC}$ exposure.
\end{abstract}

Key words: Chronic intermittent cold exposure, cold adaptation, cold inducible RNA binding protein, reactive oxidative stress, thioredoxin

\section{Introduction}

Cold stress, especially mild cold stress, occurs in our occupational or leisure time activities every day. This type of cold stress usually induces a condition of cold adaptation, reaching a new equilibrium after changing the level of stress hormones and other physiological responses. The establishment of cold adaptation in humans and animals is currently considered to be a protective mechanism against strong cold stress injury. The primary changes during cold adaptation include an increase in resting metabolic rate (RMR), leading to an increase in energy generation for maintenance of body temperature [1]. Studies have indicated that cold exposure results in an elevation of metabolic rate in mammals [2-4], an imbalance in the antioxidant defense system, increased reactive oxygen species production and oxidative stress (OS). To adapt to environmental temperature shifts, organisms have developed sophisticated strategies [5-8]. Cold-related changes in the brain and other organs are associated with increased oxidative stress and antioxidant defenses [9]. The intracellular redox balance is maintained by reactive oxygen species (ROS)-scavenger systems, mainly represented by the glutathione and the thioredoxin (TRX) systems [10]. 
Cold stress has been reported to sensitize the neuro-immune reactivity in the rat brain [11], and enhanced the metabolism rate of cold sensitive organs like liver, skeletal muscle, BAT and heart.

The molecular mechanisms underlying these responses to cold exposure in mammalian cells are just beginning to be understood [12-13]. During cold adaptation, the level of cold-inducible RNA binding protein (Cirbp, also known as Rh18P) increases in rat brains after cold exposure [14]. Cirbp, containing a consensus sequence of RNA-binding domain (CS-RBD) and a glycine-rich domain, is the first mild cold-shock protein identified in mammals [14] and is induced by mild hypothermia. Studies in vivo and in vitro have shown that Cirbp has neuroprotective effects [15-16], cytoprotective effects [17] and regulates neural development [18-20]. By now, Cirbp has been identified in humans [12], rats [30], mice [21-22], Mexican axolotls [23], bull frogs [24], Xenopus laevis [18, 25-26], and Anabaena variabilis [27]. In mammals, induction of Cirbp has been shown to correlate with an increase in the levels of antioxidant mRNAs that encode proteins involved in counteracting the effects of ROS, for example antioxidant enzymes including TRX [15, 28-29]. TRX is a ubiquitous multifunctional protein that has regulatory roles in cytoprotective activities through quenching ROS [30].

Chronic intermittent cold (CIC) exposure has been used in laboratories to generate animal models for cold stress studies [11,31-34], and previous studies mainly focused on body's thermogenesis and energy metabolism. To the authors' knowledge, currently no report is available regarding the effect of CIC exposure on oxidative damage and specific molecular changes in different tissues of rats. Therefore, we generated a cold adaptation model by exposing rats to CIC stress for 14 consecutive days, and studied changes of body temperature, ATP generation, oxidative damage and the expressions of Cirbp and TRX in various tissues of rats.

\section{Materials and methods}

\section{Experimental animals}

Five-week-old male Spargue-Dawley rats (SD rats), weighing 180-220 g, obtained from the animal center of the Fourth Military Medical University, Xi' an, China, were used in these studies. The rats were group-housed in plastic cages, maintained on a 12/12 $\mathrm{h}$ light/dark cycle and were allowed free access to food and water. After a week of acclimatization, rats were single-housed and randomly assigned to two groups (20 rats per group): control group and chronic intermittent cold (CIC) stress group. Animal care and all experimental procedures in this study were con- ducted in accordance with the guidelines of Chinese laws for animal protection and approved by the local animal care and use committee.

\section{Chronic intermittent cold stress}

Rats in the CIC stress group were treated as described previously [34]. In brief, rats were transported in their home cages, with food, water and bedding into a temperature-controlled chamber and exposed to $4^{\circ} \mathrm{C}$ for $6 \mathrm{~h}$ and returned to the housing facility for 14 consecutive days (from 10:00 am to 16:00 pm). Rats in the control group and CIC group were single-housed in a metal cage $(20 \times 20 \times 18 \mathrm{~cm})$ during this period.

\section{Measurement of rectal temperature, body weight and BAT}

Rectal temperatures of rats in CIC stress and control groups were measured at the time points before and after cold exposure using a digital laboratory thermometer (DNP-01, China). Briefly, rats were placed on a hard surface, and then the probe of a digital thermometer was inserted into rat's rectum. The temperature was recorded after it stabilized. Rats were returned to their housing facility where food and water were freely available. Control rats remained in their home chamber throughout the treatment period.

Rat body weight and the weight of BAT isolated from rats after 14 days of CIC stress were measured with a weighing scale.

\section{ROS detection}

ROS production was quantified by using the DCFH-DA method [35]. DCFH-DA can be converted to DCF by ROS-dependent oxidation, therefore acting as a direct indicator of oxidative stress. Tissues were taken from rats immediately after the last cold exposure on day 14, and then homogenized with ice-cold lysis buffer (100mmol/L phosphate buffer). The lysates were centrifuged at $1,000 \mathrm{~g}$ for $10 \mathrm{~min}$ at $4^{\circ} \mathrm{C}$, and the supernatants were transferred to fresh tubes. $190 \mu \mathrm{L}$ of supernatant were mixed with $10 \mu \mathrm{L}$ of 1mmol/L DCFH-DA (ROS Assay Kit, Nanjing Jiancheng, Jiangsu, China) or $0.01 \mathrm{~mol} / \mathrm{L}$ PBS in a 96-well plate and incubated at $37^{\circ} \mathrm{C}$ for $30 \mathrm{~min}$ in dark. Fluorescence signals were monitored at an excitation wavelength of $485 \mathrm{~nm}$ and an emission wavelength of $538 \mathrm{~nm}$ by multimodel plate reader (Infinite 200, Tecan, Männedorf, Switzerland), and then normalized by protein concentration and expressed as fluorescence intensity/mg protein.

\section{Measurement of ATP content}

The ATP levels of hippocampus, cortex, cerebellum, liver, heart, muscle and BAT was detected 
using an ATP Assay Kit (Beyotime, Jiangsu, China) as previously described [36]. Tissues were weighed (approx. $50 \mathrm{mg}$ ) and homogenized with pre-cooled extractant. After centrifugation at $12,000 \mathrm{~g}$ for 10 minutes at $4^{\circ} \mathrm{C}$, ATP was extracted using $1.5 \%$ trichloroacetic acid and supernatants were then neutralized with 1:150 Tris-acetate [pH 7.85]. $100 \mu \mathrm{l}$ working solution was added to each well containing a $10 \mu 1$ of diluted sample. The luciferase activity was immediately evaluated by multimodel plate reader. ATP content was determined by a concurrent standard curve and then normalized by protein concentration and expressed as $\mu \mathrm{mol} / \mathrm{mg}$ protein.

\section{RNA isolation, reverse transcription and quantitative real-time PCR}

Hippocampus, cortex, cerebellum, liver, heart, muscle and BAT were dissolved in TRIZOL ${ }^{\circledR}$ Reagent (Invitrogen, Barcelona, Spain) and RNA was extracted by following the manufacturer's instructions. Total RNA concentration was determined by measuring the absorbance at 260 and $280 \mathrm{~nm}$ using a UV-vis spectrophotometer (Nano 2000E Healthcare Japan, Tokyo, Japan). RNA (500ng) was reversely transcribed to cDNA using a One-Step cDNA Reverse Transcription Kit with RNase Inhibitor (DRR036A, TAKARA, Japan) according to the manufacturer's instructions. Quantitative real-time PCR was performed on an Applied Biosystems StepOne 7500 Fast Real-time PCR System. Samples were prepared using the SYBR Green PCR Master Mix (DRR041A, TAKARA, Japan) with a total volume of $10 \mu \mathrm{L}$, and were run for a total of 40 cycles with an annealing temperature of $60^{\circ} \mathrm{C}$. Each experiment was repeated at least 3 times. The sequences of primer sets used in this study were listed below:

Rat Cirbp: 5' - AGTCCCGGAGTGGTGGTTA-3' (forward) and 5' - AGGACGATCTGGACGCAGAG-3' (reverse)

Rat actin: 5'-GGAGATTACTGCCCTGGCTC CTA-3' (forward) and 5'-GACTCATCGTACTCC TGCTTGCTG-3' (reverse).

\section{Immunofluorescent staining}

After cold exposure on day 14, rats were immediately anaesthetized with $2 \%$ pentobarbital, and then perfused transcardially with NS followed by ice-cold $4 \%$ paraformaldehyde in PBS. Tissues were removed and postfixed for 2 days at $4^{\circ} \mathrm{C}$ in $25 \%$ sucrose $/ 4 \%$ paraformaldehyde. 16-mm-thick coronal sections were cut and mounted on gelatin coated slides throughout the hippocampus on a freezing microtome. Slides were stored at $-80^{\circ} \mathrm{C}$. Before staining, slides were warmed at room temperature for 30 minutes, rinsed in PBS for 3 times, and then incubated with normal serum for 30 minutes. The primary antibodies used include mouse anti- $\beta$-tubulin (Sigma-Aldrich, Missouri, USA; a widely used marker for muscle cell), rabbit anti-Cirbp (RN032P, Ribonomics, USA), and mouse anti-NeuN (MAB377, Millipore; a widely used neuronal marker). After incubated with the primary antibodies at $4{ }^{\circ} \mathrm{C}$ overnight, the slides were rinsed in PBS for 3 times, then incubated with fluorescein isothiocyanate (FITC)- or rhodamine-conjugated secondary antibodies for $1 \mathrm{~h}$. Slides were kept from light starting from this step to the end by covering slides with aluminum foil. Then slides were stained with Hoechst 33342 (Beyotime, Jiangsu, China; a widely used marker for nucleus) for $30 \mathrm{~min}$ at room temperature and rinsed in PBS for 3 times. Finally, slides were coverslipped with VECTASHIELD1 Mounting Medium (VECTOR, USA) and observed on a BX51FL fluorescence microscope (BX51FL, Olympus, Tokyo, Japan).

\section{Western blotting}

Following the termination of CIC stress, rats were anaesthetized with $2 \%$ pentobarbital. Tissues were rapidly removed on ice, and stored in aliquots at $-80^{\circ} \mathrm{C}$. For gel electrophoresis and Western blotting, frozen rat tissues were homogenized with ice-cold lysis buffer containing $20 \mathrm{mM}$ Tris- $\mathrm{HCl}, \mathrm{pH} 7.5,1 \mathrm{mM}$ EDTA, $5 \mathrm{mM} \mathrm{MgCl2}, 1 \mathrm{mM}$ dithiothreitol (DTT), 20 $\mu \mathrm{g} / \mathrm{ml}$ aprotinin, and $1 \mathrm{mM}$ phenylmethylsulfonyl fluoride (PMSF). The homogenates was centrifuged at $12500 \mathrm{rpm}$ for $15 \mathrm{~min}$ at $4^{\circ} \mathrm{C}$. The protein concentration was determined using a BCA kit (BCA Protein Assay Kit, Pierce, USA). Aliquots from supernatant containing 50-100 $\mu \mathrm{g}$ proteins were mixed with equal volume of $2 \times$ loading buffer. All the steps of protein preparation were carried out at $4^{\circ} \mathrm{C}$. Samples were boiled at $100^{\circ} \mathrm{C}$ for $5 \mathrm{~min}$ and subjected to $12 \%$ SDS-PAGE and transferred to a polyvinylidene difluoride membrane (PVDF, Millipore, Darmstadt, Germany) using an electrophoretic transfer system (Bio-Rad, Hercules, CA). The membranes were blocked in blocking buffer containing 5\% non-fat dry milk with gentle shaking to prevent non-specific binding at room temperature for $30 \mathrm{~min}$, and then incubated with primary antibodies at $4^{\circ} \mathrm{C}$ overnight. The membranes were washed in TBST $(50 \mathrm{mmol} / \mathrm{L}$ Tris-Cl, pH 7.6, $150 \mathrm{mmol} / \mathrm{L} \mathrm{NaCl}, 0.1 \%$ Tween 20) for $30 \mathrm{~min}$ and incubated with appropriate horseradish peroxidase-conjugated (HRP) conjugated secondary antibody (1:1000 dilution) for $2 \mathrm{~h}$ at room temperature. The membranes were washed three times in TBST and the blots were developed with an enhanced chemiluminescence kit according to the manufacturer's instructions (Amersham Biosciences). For semi-quantitative analysis of immunoblot bands, the 
density of each band was measured with a computer imaging device and accompanying software (Bio-Rad). The background value was subtracted from all other readings.

To verify equal protein loading and transfer, the blots were probed with an anti- $\beta$-actin antibody (Sigma-Aldrich, Missouri, USA, 1:5000 dilution) and anti- $\beta$-tubulin antibody (1:1000 dilution).

\section{Statistical analysis}

All data are expressed as mean \pm SEM. and analyzed by SPSS13.0. T-tests used for comparison of two groups and repeated measures ANOVA was applied for analysis of body temperature across 14 days. Differences were considered significant at $\mathrm{p}<0.05$.

\section{Results}

\section{Establishment of a cold adaptation model}

To generate a cold adaptation model, rats were housed in a $4^{\circ} \mathrm{C}$ cold room for $6 \mathrm{~h}$ per day for 14 consecutive days. Rectal temperature was measured before and after cold exposure. As shown in Fig. 1A, rat rectal temperature declined drastically after cold exposure on the first day $(p<0.001)$. The extent of rectal temperature decline after cold exposure gradually reduced along with the increase in days of CIC exposure, and was negligible after 14 consecutive days
A

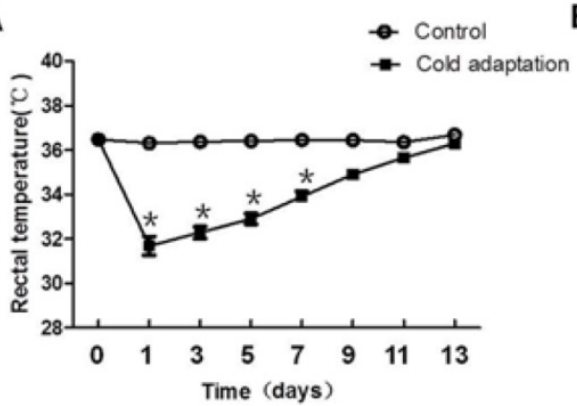

C

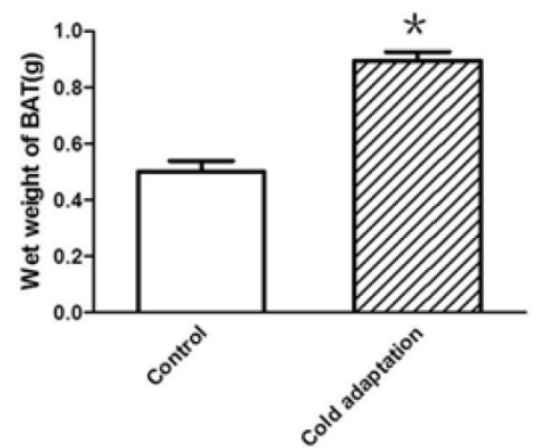

B

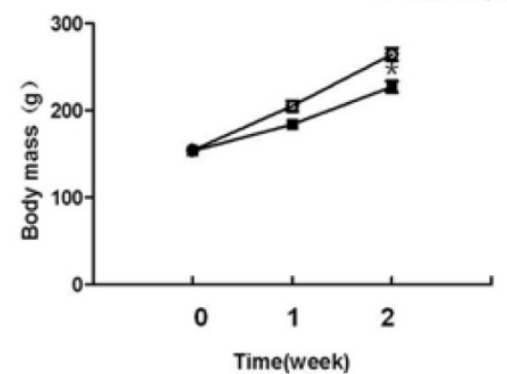

D

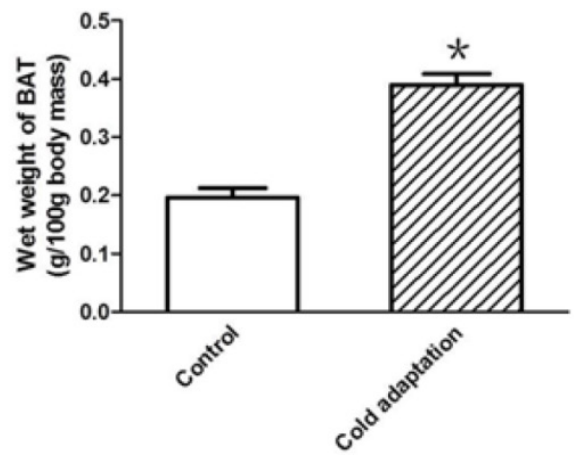

Fig. 1. Establishment of a cold adaptation model by $\mathrm{CIC}$ stress. (A) The change of rectal temperature in SD rats during cold exposure. (B) The change of body mass in SD rats during cold exposure. (C) Mean wet BAT mass and (D) mean wet weight of $100 \mathrm{~g}$ body mass in control and cold adaptation groups (14 days). * depicts significant difference between cold adaptation and the control groups $(n=10$, mean \pm SEM, $p<$ $0.05)$. (day 11, $p=0.059$; day $13, p=0.107$ ), suggesting establishment of a cold adaptation. In addition to rectal temperature, we also measured body weight and BAT weight of rats in the control and CIC stress groups. Compared to rats in the control group, rats in CIC stress group showed a lower average body weight after one consecutive week of CIC stress (Fig. 1B). The average body weight of rats declined further after two consecutive weeks of cold exposure (Fig. 1B, $t=3.923$, $\mathrm{DF}=18, p<0.001$ ), reducing to $86 \%$ of the body weight of control rats. In contrast, BAT weight was increased in rats after 2 weeks of cold exposure as indicated by an increase of $78.7 \%$ in wet mass of BAT (Fig. $1 \mathrm{C}, \mathrm{t}=3.651, \mathrm{DF}=18, p=0.002$ ) and $98.1 \%$ in BAT weight of $100 \mathrm{~g}$ body mass (Fig.1D, $\mathrm{t}=5.289, \mathrm{DF}=$ $18, p<0.001)$, with an about one-fold increase.

\section{CIC stress increased ATP and ROS generation in rat organs}

Cold exposure induces an increase in energy generation that correlates with an elevation of ROS production. Mitochondria are the principal site for the generation of cellular ATP by oxidative phosphorylation. Accompanied by this procession, some leakage of the high-energy electrons in the respiratory chain gives rise to reactive oxygen species. To examine the effect of CIC stress on energy generation, we detected the levels of ATP and ROS in a variety of organs. As shown in Fig. 2, the levels of both ATP (Fig. 2A) and ROS (Fig. 2B) were increased in organs including the hippocampus $(\mathrm{t}=$ 4.792, $\mathrm{DF}=8, p=0.001 ; \mathrm{t}=$ 3.246, $\mathrm{DF}=8, p=0.012$, respectively), cortex $(\mathrm{t}=3.158, \mathrm{DF}=8$, $p=0.013 ; \mathrm{t}=3.952, \mathrm{DF}=8, p=$ $0.004)$ and cerebellum $(t=3.125$, $\mathrm{DF}=8, p=0.014 ; \mathrm{t}=3.24, \mathrm{DF}=$ $8, p=0.012$ ) of rat brains, livers $(\mathrm{t}=3.092, \mathrm{DF}=8, p=0.015 ; \mathrm{t}=$ 4.471, DF $=8, p=0.002$ ), hearts $(\mathrm{t}=4.459, \mathrm{DF}=8, p=0.002 ; \mathrm{t}=$ 2.827, $\mathrm{DF}=8, p=0.022)$, muscles $(\mathrm{t}=3.664, \mathrm{DF}=8, p=0.006$; $\mathrm{t}=3.103, \mathrm{DF}=8, p=0.015)$ and BATs $(\mathrm{t}=3.744, \mathrm{DF}=8, p=$ $0.006 ; \mathrm{t}=3.048, \mathrm{DF}=8, p=$ 0.016 ) isolated from rats receiving 14 consecutive days of CIC stress as compared to those in organs of rats in the control group. 


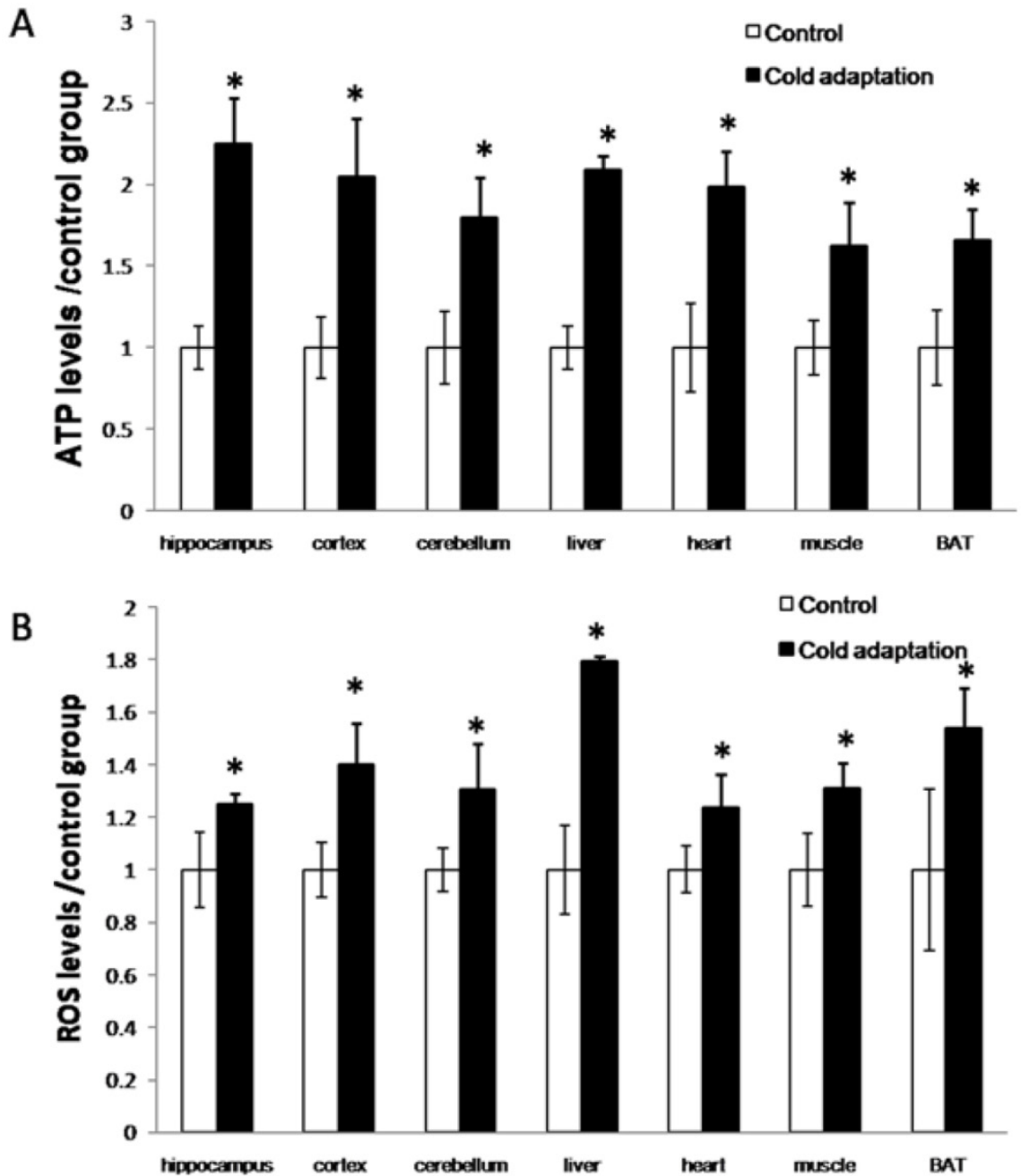

Fig.2. Increase of ATP and ROS levels after cold adaptation in rat tissues. (A) The levels of ATP in the indicated tissues were measured by luminescence using an ATP detection kit. (B) The levels of ROS in the indicated tissues were assessed by Fluorescence microscope after DCFH-DA staining. * depicts significant difference between cold adaptation and the control groups $(n=5$, mean \pm SEM, $p<0.05)$.

\section{CIC stress induced Cirbp expression}

Cirbp can be induced by mild cold stress, and has a cryoprotective effect. To investigate whether CIC stress can induce the expression of Cirbp, we first measured the levels of Cirbp mRNA in tissues from rat in CIC stress and control groups by RT-PCR. As shown in Fig. 3, the levels of Cirbp mRNA were higher in the tissues including hippocampus $(\mathrm{t}=$ 2.958, $\mathrm{DF}=8, p=0.018)$, cortex $(\mathrm{t}=4.238, \mathrm{DF}=8, p=$ $0.003)$ and cerebellum $(\mathrm{t}=3.667, \mathrm{DF}=8, p=0.006)$, heart $(\mathrm{t}=3.097, \mathrm{DF}=8, p=0.015)$, liver $(\mathrm{t}=2.276, \mathrm{DF}=$ $8, p=0.052)$, BAT $(\mathrm{t}=4.173, \mathrm{DF}=8, p=0.003)$, and muscle $(\mathrm{t}=2.260, \mathrm{DF}=8, p=0.054)$ isolated from rats receiving 14 consecutive days of CIC stress than those in tissues isolated from control rats. Of note, the increases of Cirbp mRNA in the liver and muscle were not as dramatic as those of other tissues (Fig. 3).

We next assessed the expression levels of Cirbp protein in tissues prepared from rats in CIC stress and the control groups by Western blotting. In line with the increase in Cirbp mRNA, CIC stress led to an increase in the levels of Cirbp protein (Fig. 4) in hippocampus $(\mathrm{t}=3.404, \mathrm{DF}=8, p=0.009)$, cortex $(\mathrm{t}=3.110$, $\mathrm{DF}=8, p=0.014)$ and cerebellum $(\mathrm{t}=2.875, \mathrm{DF}=8, p=$ 0.021 ) (Fig. 4A), liver $(\mathrm{t}=2.851, \mathrm{DF}=8, p=0.021)$ and BAT $(\mathrm{t}=3.058, \mathrm{DF}=8, p=0.016)$ (Fig. 4B), heart $(\mathrm{t}=$ 2.932, $\mathrm{DF}=8, p=0.019)$ and muscle $(\mathrm{t}=3.032, \mathrm{DF}=8$, $p=0.016$ ) (Fig. 4C). Of note, the levels of Cirbp protein were increased in the livers and muscles of CIC-stressed rats although the increases in Cirbp mRNA in these two organs were not statistically significant. This inconsistence may be due to post-transcriptional modification or other unknown mechanisms.

To observe the subcelluar localization and the difference of Cirbp protein expression between tissues from CIC stress and the control groups, we performed immunofluorescent staining. Tissue sections were stained with anti-Cirbp coupled with anti-NeuN (for brain) and anti-tubulin (for heart and muscle). Im- 
munofluorescent analysis of rat brain sections indicated that expression of Cirbp protein was located in nucleus and cytoplasm, mainly in the perinuclear region, and increased significantly after 14 days of CIC exposure as compared with the control group (Fig. 5A-C). In heart (Fig. 5D), muscle (Fig. 5E), BAT (Fig. 5F) and liver (Fig. 5G) isolated from CIC-stressed rats, the levels of Cirbp protein were also increased, with a main location in cytoplasm. Together, these data indicate that CIC stress can induce Cirbp expression, which may facilitate the process of cold adaptation.

\section{CIC stress induced TRX expression}

Cirbp induction has been shown to correlate with an increase in TRX expression. We then determined the levels of TRX protein in tissues isolated from CIC-stressed and control rats. In agreement with high levels of Cirbp, the expression of TRX was increased in hippocampus $(\mathrm{t}=3.102, \mathrm{DF}=8, p=0.015)$, cortex $(\mathrm{t}=3.450, \mathrm{DF}=8, p=0.009)$, cerebellum $(\mathrm{t}=$ 3.364, $\mathrm{DF}=8, p=0.01)$ and liver $(\mathrm{t}=3.029, \mathrm{DF}=8, p=$ 0.016) (Fig. 6A), BAT $(\mathrm{t}=2.926, \mathrm{DF}=8, p=0.019$ ) (Fig. $6 \mathrm{~B})$, heart $(\mathrm{t}=3.285, \mathrm{DF}=8, p=0.011)$ and muscle $(\mathrm{t}=$ 2.748, $\mathrm{DF}=8, p=0.025$ ) (Fig. 6C) prepared from CIC stressed rats.

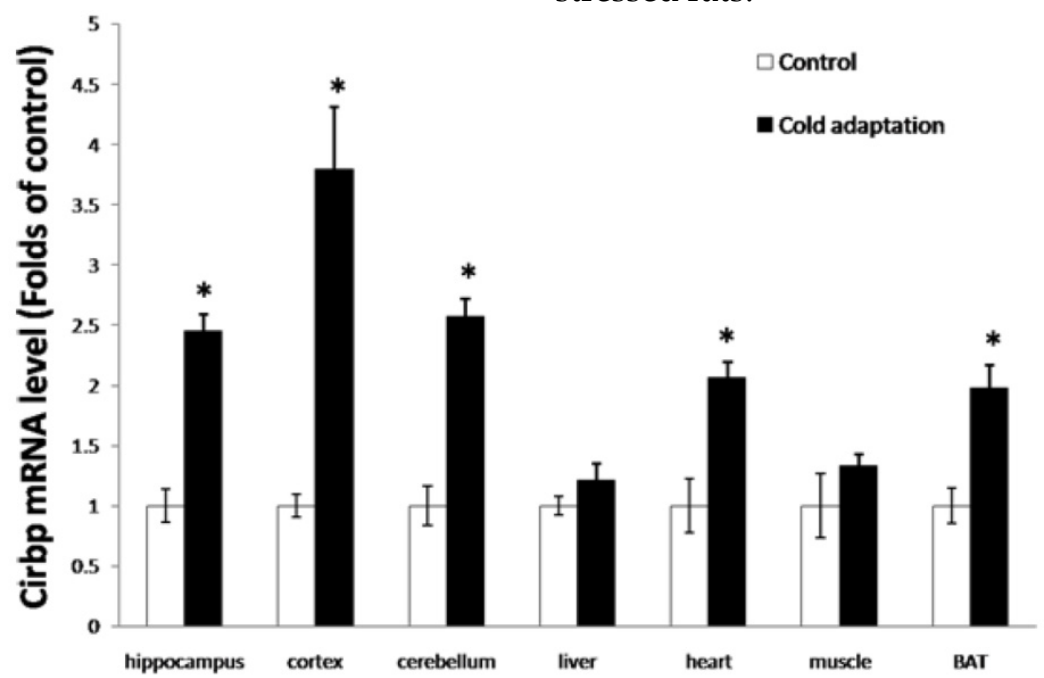

Fig.3. Increase of Cirbp mRNA in tissues isolated from CIC-stressed rats. The levels of Cirbp mRNA in hippocampus, cortex, cerebellum, heart, liver, BAT and muscle isolated from $\mathrm{ClC}$-stressed and control rats were assesses by quantitative RT-PCR $(n=5$, mean \pm SEM). * depicts significant difference between cold adaptation and the control groups $(p<0.05)$.
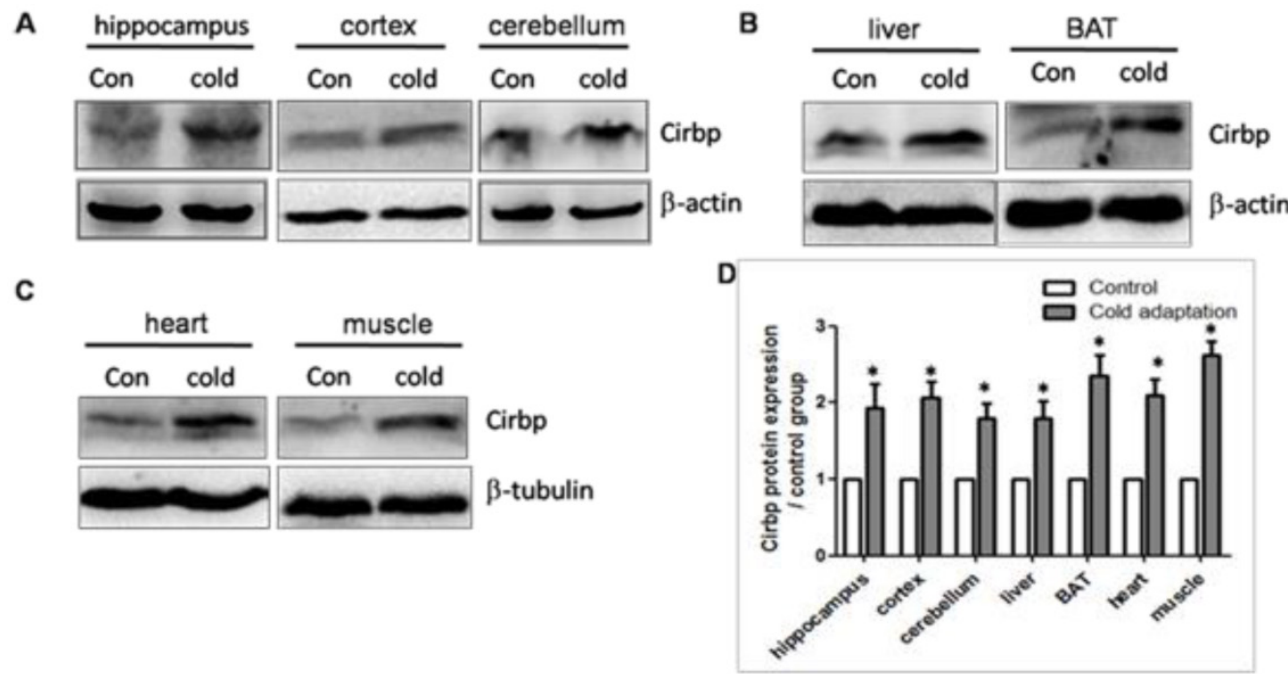

Fig. 4. Increase of Cirbp protein in tissues isolated from CIC-stressed rats. The levels of Cirbp protein in hippocampus, cortex, cerebellum (A), liver and BAT (B), heart and muscle (C) isolated from control and CIC-stressed rats were determined by Western blotting. $\beta$-actin or $\beta$-tubulin was used as a loading control. The relative amounts of Cirbp were quantitated and normalized by $\beta$-actin or $\beta$-tubulin (D). * depicts significant difference between cold adaptation and the control groups $(n=5$, mean \pm SEM, $p<0.05)$. 


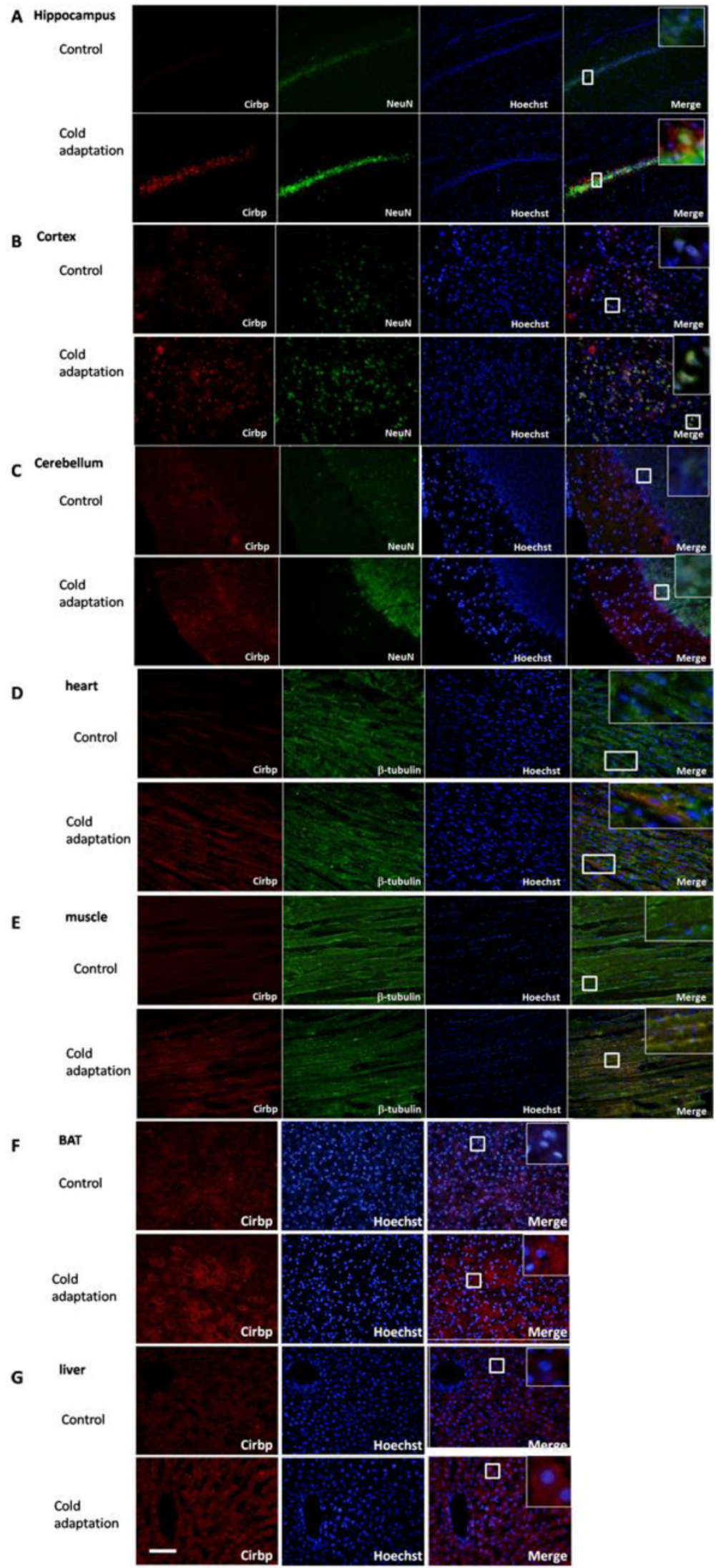

Fig. 5. Immunofluorescence staining of Cirbp in hippocampus (A), cortex (B), cerebellum (C), heart (D), muscle (E), BAT (F)and liver (G). Cirbp and tissue markers NeuN (brain) and $\beta$-tubulin (heart and muscle) were stained with corresponding primary antibodies followed by FITC- (green for Cirbp) or rhodamine- (red for NeuN and $\beta$-tubulin) conjugated secondary antibodies. The nuclei are stained with Hoechst 33342 (blue). The staining was then observed using a fluorescent microscope. Bar "- " $=100 \mu \mathrm{m}, \mathrm{n}=3$. 
A

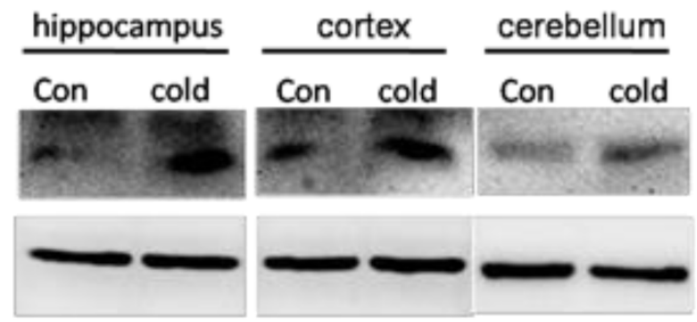

C

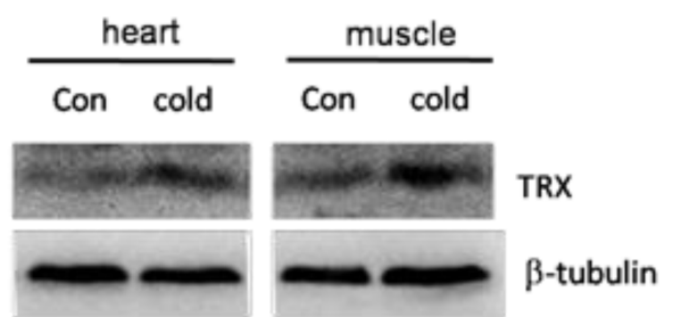

D
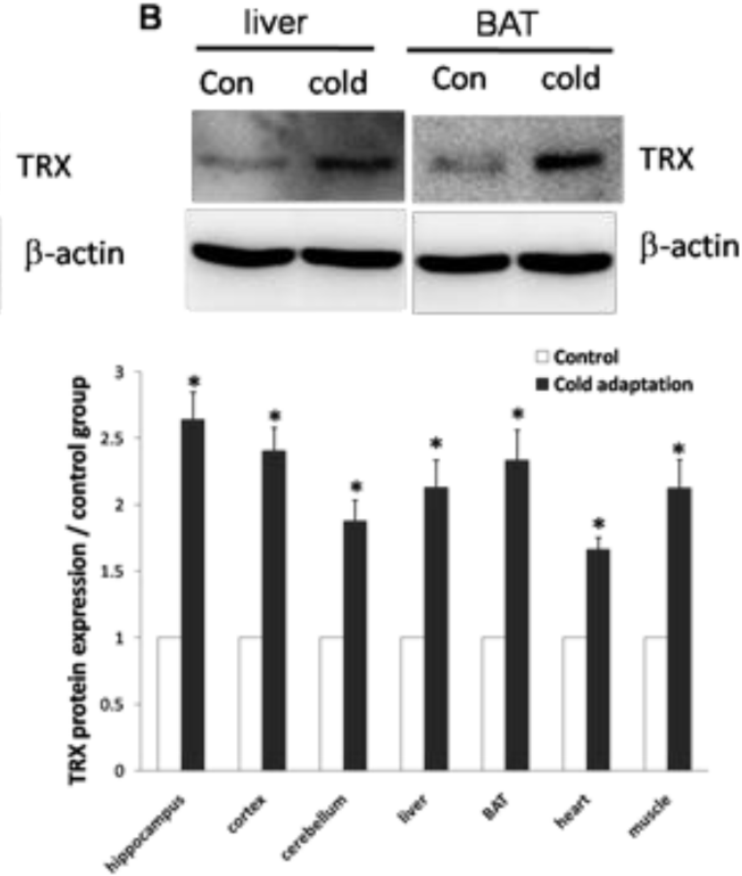

Fig. 6. Increase of TRX protein in rat tissues after CIC exposure. The levels of TRX protein in hippocampus, cortex, cerebellum (A), liver and BAT (B), heart and muscle $(C)$ isolated from control and $\mathrm{CIC}$-stressed rats were determined by Western blotting. $\beta$-actin or $\beta$-tubulin was used as a loading control. The relative amounts of TRX were quantitated and normalized by $\beta$-actin or $\beta$-tubulin (D). * depicts significant difference between cold adaptation and the control groups $(n=5$, mean \pm SEM, $p<0.05)$.

\section{Discussion}

Environmental temperature plays an important role in regulating animals' physiology and behavior. Exposure of the body to CIC is known to be a metabolic stressor and under natural conditions CIC is more often encountered intermittently rather than continuously. It is well known that a severe cold exposure causes marked whole body cooling and therefore impairs the function of organs such as central nervous system (CNS). However, studies on stress-induced changes in response to cold exposure are inconclusive. Cold adaptation induced by exposure of mild cold environment, which does not decrease core temperature markedly, may produce protective effects. Previous studies on cold adaptation mainly focused on body's thermogenesis and energy metabolism. However, the effect of chronic intermittent cold exposure on vital organs has not been fully characterized and the molecular mechanism underlying it is rarely studied.

This study demonstrates that a daily CIC exposure at $4{ }^{\circ} \mathrm{C}$ for $6 \mathrm{~h} /$ day for 2 weeks can elicit adaptations. The changes of rats' rectal temperatures were negligible after 14 consecutive days of CIC exposure (Fig.1A), indicating establishment of cold adaptation. Cold-adapted rats exhibited lower average body weight, but higher amount of BAT as compared to control rats (Fig.1B-D). Change of body weight is an important adaptive strategy for many small mammals
[25-26], which can be an increase [37], no change [38], or even a decrease [39] during cold adaptation. The variations in body mass are associated with changes in RMR, energy metabolism and other biochemical markers.

Considerable evidence indicates that mild core hypothermia directly increases heat generation, and increased heat generation is essential for keeping body temperature balance under cold stress. Either optimal BAT mass or thermogenic function is important for protecting animals against cold [40]. The facultative thermogenesis primarily occurs in the BAT and skeletal muscle [41]. BAT is the main site of facultative thermogenesis in small rodents. Cold stress has been reported to activate BAT, accompanied by macroscopic, microscopic and biochemical changes in BAT functions [42]. BAT is an important organ of nonshivering thermogenesis (NST) induced by cold [43]. It can be speculated that intact BAT might be critical for maintaining normal euthermic state of cold-adapted animals [44]. In this study, rats established cold adaptation after 14 days of CIC exposure, containing a higher average weight of BAT. These findings suggest that BAT may have an essential role for rats to establish the cold adaptation. In summary, our data support the idea that BAT may play an important role in thermoregulation in cold-adapted rats.

Exposure of cold stress leads to a higher rate of metabolism to cope with increased energy demands 
in various organs. Increase in metabolic rate relies on increased oxygen consumption, intensive oxidation of fatty acids, and is in the line with tissue structural remodeling [43]. All these processes are known to alter redox-state, i.e. concentration of ROS. To adapt cold stress, organisms enhance energy metabolism, indicating an overall increase in the capacity for ATP production accompanied by an increase of ROS generation. It is known that skeletal muscle and liver are major oxygen consumers, and their combined activities take up near $40 \%$ of cardiac oxygen delivery [45]. Meanwhile, brain and heart [46] are both require very high systemic oxygen and account for $25 \%$ and $7 \%$, respectively. Our study has been consistent with them that ATP content and ROS generation among these high oxygen consuming organs were increased significantly in cold-adapted rats as compared with control rats.

ROS increase is a hallmark of chronic neurodegenerative diseases, having been implicated as key mechanisms of chronic and neurotoxic microglial activation, particularly in the case of Parkinson's disease [47]. The high level of ROS in the brain of CIC-exposed rats may provide one potential mechanism underlying CIC stress-induced sensitization of neuro-immune reactivity in the rat brain [11].

Accordingly, increases in ROS-scavenger system, i.e. glutathione peroxidase (GSH-Px) and thioredoxin (TRX) systems activity [10]. TRX is a ubiquitous multifunctional protein that has regulatory roles in cytoprotective activities through quenching reactive oxygen species (ROS), and regulating cellular signaling and gene transcription [30]. Interestingly, ROS accumulation in cells appears to have a strong influence on cold regulation of TRX gene expression [48]. A recent study has shown that cold exposure causes oxidative stress through inhibition of TRX function in vitro [49]. Meanwhile, another study presents that low doses of ROS protect cells from apoptosis by increasing TRX expression [50]. In this study, we found that CIC stress leads to an up-regulation of TRX in the brain, liver, heart, muscle, BAT.

Cirbp has been shown to be induced by mild cold and other stresses including UV radiation, hypoxia and osmotic pressure. Our results showed that the expression of Cirbp was significantly induced in rat organs after CIC exposure. It may provide protective effects against brain damage, cardiac arrest and liver injury under mild hypothermia $\left(32-33^{\circ} \mathrm{C}\right)$. The mechanism underlying this may related to the activation of c-Jun N-terminal kinase and Akt, which are involved in ROS accumulation, decreased oxidized protein levels and increased antioxidant levels [51]. Cirbp's antioxidant effects may thoroughly regulate the expression of antioxidant proteins that are in- volved in counteracting the effects of ROS $[15,28]$. Previous study has shown that the expression of TRX can be affected by Cirbp under hypothermia [15, 28-29]. In this study, the increased expression level of TRX consistant with Cirbp, supporting a link between these two proteins. Although Cirbp has been reported to possess cytoprotective effects [15], the underlying mechanisms remain incompletely understood. In addition, its role in adaptation to CIC stress is not clear. These interesting questions are currently under investigation in our group.

In summary, this paper systematic studied the change of Cirbp in CIC stress. The data showed that CIC stress increases the generation of ATP and ROS, induces the expression of Cribp and TRX in the brains, livers, hearts, muscles and BATs of rats. The results provide some new information to understand the physiological role of Cirbp in cold adaptation.

\section{Abbreviations}

CIC: chronic intermittent cold; BAT: brown adipose tissue; ROS: reactive oxygen species; Cirbp: cold-inducible RNA binding protein; TRX: thioredoxin; RMR: resting metabolic rate; OS: oxidative stress; CS-RBD: consensus sequence of RNA-binding domain; DTT: dithiothreitol; PMSF: phenylmethylsulfonyl fluoride; HRP: horseradish peroxidase-conjugated; CNS: central nerves system; NST: nonshivering thermogenesis.

\section{Acknowledgements}

This work was supported by Key of National Natural Science Foundation of China: (\#81330045); National Natural Science Foundation of China (\#80330045, \#81172621); National Major Scientific and Technological Special Project for "Significant New Drugs Development” (\#2011ZXJ09201-023); This work was also supported by Program for Changjiang Scholars (T2011153) and Innovative Research Team in University (PCSIRT).

\section{Competing Interests}

There are no financial or other interests with regard to the paper that represent a conflict of interest.

\section{References}

1. Young BA. Cold stress as it affects animal production. J Anim Sci. 1981; 52: 154-63.

2. Pilcher JJ, Nadler E, and Busch C. Effects of hot and cold temperature exposure on performance: a meta-analytic review. Ergonomics. 2002; 45: 682-98.

3. Selman C, Grune T, Stolzing A, Jakstadt M, McLaren JS, and Speakman JR. The consequences of acute cold exposure on protein oxidation and proteasome activity in short-tailed field voles, microtus agrestis. Free Radic Biol Med. 2002; 33: 259-65.

4. Venditti P, De Rosa R, Portero-Otin M, Pamplona R, and Di Meo S. Cold-induced hyperthyroidism produces oxidative damage in rat tissues and increases susceptibility to oxidants. Int J Biochem Cell Biol. 2004; 36: 1319-31.

5. Rao PN and Engelberg J. Hela Cells: Effects of Temperature on the Life Cycle. Science. 1965; 148: 1092-4. 
6. Burdon RH. Thermotolerance and the heat shock proteins. Symp Soc Exp Biol. 1987; 41: 269-83.

7. Burdon RH, Gill VM, and Rice-Evans C. Oxidative stress and heat shock protein induction in human cells. Free Radic Res Commun. 1987; 3: 129-39.

8. Aloia RC and Raison JK. Membrane function in mammalian hibernation. Biochim Biophys Acta. 1989; 988: 123-46.

9. Sakurai T, Yada N, Watanabe T, et al. Cold-inducible RNA-binding protein promotes the development of liver cancer. Cancer Sci. 2015.

10. Yamawaki H and Berk BC. Thioredoxin: a multifunctional antioxidant enzyme in kidney, heart and vessels. Curr Opin Nephrol Hypertens. 2005; 14: 149-53.

11. Girotti M, Donegan JJ, and Morilak DA. Chronic intermittent cold stress sensitizes neuro-immune reactivity in the rat brain. Psychoneuroendocrinology. 2011; 36: 1164-74.

12. Glofcheski DJ, Borrelli MJ, Stafford DM, and Kruuv J. Induction of tolerance to hypothermia and hyperthermia by a common mechanism in mammalian cells. J Cell Physiol. 1993; 156: 104-11.

13. Matz JM, Blake MJ, Tatelman HM, Lavoi KP, and Holbrook NJ. Characterization and regulation of cold-induced heat shock protein expression in mouse brown adipose tissue. Am J Physiol. 1995; 269: R38-47.

14. Nishiyama $\mathrm{H}$, Higashitsuji $\mathrm{H}$, Yokoi $\mathrm{H}$, et al. Cloning and characterization of human CIRP (cold-inducible RNA-binding protein) cDNA and chromosomal assignment of the gene. Gene. 1997; 204: 115-20.

15. Liu A, Zhang Z, Li A, and Xue J. Effects of hypothermia and cerebral ischemia on cold-inducible RNA-binding protein mRNA expression in rat brain. Brain Res. 2010; 1347: 104-10.

16. Liu J, Xue J, Zhang H, et al. Cloning, expression, and purification of cold inducible RNA-binding protein and its neuroprotective mechanism of action. Brain Res. 2015; 1597: 189-95.

17. Sakurai $\mathrm{T}$, Itoh $\mathrm{K}$, Higashitsuji $\mathrm{H}$, et al. Cirp protects against tumor necrosis factor-alpha-induced apoptosis via activation of extracellular signal-regulated kinase. Biochim Biophys Acta. 2006; 1763: 290-5.

18. Peng $\mathrm{Y}, \mathrm{Kok} \mathrm{KH}, \mathrm{Xu} \mathrm{RH}$, et al. Maternal cold inducible RNA binding protein is required for embryonic kidney formation in Xenopus laevis. FEBS Lett. 2000; 482: $37-43$.

19. van Venrooy S, Fichtner D, Kunz M, Wedlich D, and Gradl D. Cold-inducible RNA binding protein (CIRP), a novel XTcf-3 specific target gene regulates neural development in Xenopus. BMC Dev Biol. 2008; 8: 77.

20. Masuda $\mathrm{T}$, Itoh $\mathrm{K}$, Higashitsuij $\mathrm{H}$, et al. Cold-inducible RNA-binding protein (Cirp) interacts with Dyrk1b/Mirk and promotes proliferation of immature male germ cells in mice. Proc Natl Acad Sci U S A. 2012; 109: 10885-90.

21. Nishiyama H, Itoh K, Kaneko Y, Kishishita M, Yoshida O, and Fujita J. A glycine-rich RNA-binding protein mediating cold-inducible suppression of mammalian cell growth. J Cell Biol. 1997; 137: 899-908.

22. Xia Z, Jiang $K$, Liu $T$, Zheng $H$, Liu $X$, and Zheng $X$. The protective effect of Cold-inducible RNA-binding protein (CIRP) on testicular torsion/detorsion: an experimental study in mice. J Pediatr Surg. 2013; 48: 2140-7.

23. Bhatia R, Dube DK, Gaur A, et al. Expression of axolotl RNA-binding protein during development of the Mexican axolotl. Cell Tissue Res. 1999· 297: 283-90.

24. Saito T, Sugimoto K, Adachi $\mathrm{Y}, \mathrm{Wu} \mathrm{Q}$, and Mori KJ. Cloning and characterization of amphibian cold inducible RNA-binding protein. Comp Biochem Physiol B Biochem Mol Biol. 2000; 125: 237-45.

25. Uochi $\mathrm{T}$ and Asashima M. XCIRP (Xenopus homolog of cold-inducible RNA-binding protein) is expressed transiently in developing pronephros and neural tissue. Gene. 1998; 211: 245-50.

26. Aoki $\mathrm{K}$, Matsumoto $\mathrm{K}$, and Tsujimoto $\mathrm{M}$. Xenopus cold-inducible RNA-binding protein 2 interacts with ElrA, the Xenopus homolog of HuR, and inhibits deadenylation of specific mRNAs. J Biol Chem. 2003; 278: 48491-7.

27. Sato N. A family of cold-regulated RNA-binding protein genes in the cyanobacterium Anabaena variabilis M3. Nucleic Acids Res. 1995; 23: 2161-7.

28. Valic Z, Palada I, Bakovic D, Valic M, Mardesic-Brakus S, and Dujic Z. Muscle oxygen supply during cold face immersion in breath-hold divers and controls. Aviat Space Environ Med. 2006; 77: 1224-9.

29. Li S, Zhang Z, Xue J, Liu A, and Zhang H. Cold-inducible RNA binding protein inhibits $\mathrm{H}(2) \mathrm{O}(2)$-induced apoptosis in rat cortical neurons. Brain Res. 2012; 1441: 47-52.

30. Tanaka T, Kondo S, Iwasa Y, Hiai H, and Toyokuni S. Expression of stress-response and cell proliferation genes in renal cell carcinoma induced by oxidative stress. Am J Pathol. 2000; 156: 2149-57.

31. Dorfman $\mathrm{M}$, Ramirez VD, Stener-Victorin $\mathrm{E}$, and Lara $\mathrm{HE}$ Chronic-intermittent cold stress in rats induces selective ovarian insulin resistance. Biol Reprod. 2009; 80: 264-71.

32. Pardon $\mathrm{MC}, \mathrm{Ma} \mathrm{S}$, and Morilak DA. Chronic cold stress sensitizes brain noradrenergic reactivity and noradrenergic facilitation of the HPA stress response in Wistar Kyoto rats. Brain Res. 2003; 971: 55-65

33. Girotti M, Donegan JJ, and Morilak DA. Influence of hypothalamic IL-6/gp130 receptor signaling on the HPA axis response to chronic stress. Psychoneuroendocrinology. 2013; 38: 1158-69.

34. Lapiz-Bluhm MD, Soto-Pina AE, Hensler JG, and Morilak DA. Chronic intermittent cold stress and serotonin depletion induce deficits of reversal learning in an attentional set-shifting test in rats. Psychopharmacology (Berl). 2009; 202: 329-41.

35. Lawler JM, Song W, and Demaree SR. Hindlimb unloading increases oxidative stress and disrupts antioxidant capacity in skeletal muscle. Free Radic Biol Med. 2003; 35: 9-16.
36. He $\mathrm{S}$, Atkinson $\mathrm{C}$, Qiao $\mathrm{F}$, Cianflone $\mathrm{K}$, Chen $\mathrm{X}$, and Tomlinson $\mathrm{S}$, A complement-dependent balance between hepatic ischemia/reperfusion injury and liver regeneration in mice. J Clin Invest. 2009; 119: 2304-16.

37. Wang $\mathrm{Z}, \mathrm{Li} \mathrm{Q}$, and Sun $\mathrm{R}$. The characteristics of nonshivering thermogenesis and cellular respiration in the tree shrews. Zoological research/" Dong wu xue yan jiu" bian ji wei yuan hui bian ji. 1994; 16: 239-246.

38. REYNOLDS P and LAVIGNE D. Photoperiodic effects on body size end energetics of the collared lemming, Dicrostonyx groenlandicus. Canadian journal of zoology. 1988; 66: 835-841.

39. Merritt JF. Seasonal thermogenesis and changes in body mass of masked shrews, Sorex cinereus. Journal of Mammalogy. 1995; 76: 1020-1035.

40. de Jesus LA, Carvalho SD, Ribeiro MO, et al. The type 2 iodothyronine deiodinase is essential for adaptive thermogenesis in brown adipose tissue. J Clin Invest. 2001; 108: 1379-85.

41. Foster DO and Frydman ML. Tissue distribution of cold-induced thermogenesis in conscious warm- or cold-acclimated rats reevaluated from changes in tissue blood flow: the dominant role of brown adipose tissue in the replacement of shivering by nonshivering thermogenesis. Can J Physiol Pharmacol. 1979; 57: 257-70.

42. Carter EA, Bonab AA, Hamrahi V, et al. Effects of burn injury, cold stress and cutaneous wound injury on the morphology and energy metabolism of murine brown adipose tissue (BAT) in vivo. Life Sci. 2011; 89: 78-85.

43. Cannon B and Nedergaard J. Brown adipose tissue: function and physiological significance. Physiol Rev. 2004; 84: 277-359.

44. Nedergaard J, Golozoubova V, Matthias A, Asadi A, Jacobsson A, and Cannon B. UCP1: the only protein able to mediate adaptive non-shivering thermogenesis and metabolic inefficiency. Biochim Biophys Acta. 2001; 1504: $82-106$.

45. Zaninovich AA, Rebagliati I, Raices M, Ricci C, and Hagmuller K. Mitochondrial respiration in muscle and liver from cold-acclimated hypothyroid rats. J Appl Physiol. 2003; 95: 1584-90.

46. Faraci FM, Kilgore DL, Jr., and Fedde MR. Oxygen delivery to the heart and brain during hypoxia: Pekin duck vs. bar-headed goose. Am J Physiol. 1984; 247: R69-75.

47. Lull ME and Block ML. Microglial activation and chronic neurodegeneration. Neurotherapeutics. 2010; 7: 354-65.

48. Chinnusamy V, Zhu J, and Zhu JK. Cold stress regulation of gene expression in plants. Trends Plant Sci. 2007; 12: 444-51.

49. Zieger MA, Gupta MP, and Wang M. Proteomic analysis of endothelial cold-adaptation. BMC Genomics. 2011; 12: 630

50. Haendeler J, Tischler V, Hoffmann J, Zeiher AM, and Dimmeler S. Low doses of reactive oxygen species protect endothelial cells from apoptosis by increasing thioredoxin-1 expression. FEBS Lett. 2004; 577: 427-33.

51. Sakurai T, Kudo M, Watanabe T, et al. Hypothermia protects against fulminant hepatitis in mice by reducing reactive oxygen species production. Dig Dis. 2013; 31: 440-6. 